TRANSACTIONS OF THE

AMERICAN MATHEMATICAL SOCIETY

Volume 355, Number 2, Pages 593-608

S 0002-9947(02)03124-0

Article electronically published on October 4, 2002

\title{
RESOLUTIONS OF IDEALS OF QUASIUNIFORM FAT POINT SUBSCHEMES OF $\mathbf{P}^{2}$
}

\author{
BRIAN HARBOURNE, SANDEEP HOLAY, AND STEPHANIE FITCHETT
}

\begin{abstract}
The notion of a quasiuniform fat point subscheme $Z \subset \mathbf{P}^{2}$ is introduced and conjectures for the Hilbert function and minimal free resolution of the ideal $I$ defining $Z$ are put forward. In a large range of cases, it is shown that the Hilbert function conjecture implies the resolution conjecture. In addition, the main result gives the first determination of the resolution of the $m$ th symbolic power $I(m ; n)$ of an ideal defining $n$ general points of $\mathbf{P}^{2}$ when both $m$ and $n$ are large (in particular, for infinitely many $m$ for each of infinitely many $n$, and for infinitely many $n$ for every $m>2$ ). Resolutions in other cases, such as "fat points with tails", are also given. Except where an explicit exception is made, all results hold for an arbitrary algebraically closed field $k$. As an incidental result, a bound for the regularity of $I(m ; n)$ is given which is often a significant improvement on previously known bounds.
\end{abstract}

\section{INTRODUCTION}

Let $p_{1}, \ldots, p_{n} \in \mathbf{P}^{2}$ be general points; given a variety $X$, we always use " $n$ general points of $X$ " in the sense of "on an open and dense subset of $X^{n}$ ". Let $R=k\left[\mathbf{P}^{2}\right]$ be the homogeneous coordinate ring of $\mathbf{P}^{2}$, and let $P_{j}$ be the homogeneous ideal generated by all forms in $R$ vanishing at $p_{j}$. An ideal of the form $I(\mathbf{m} ; n)=P_{1}^{m_{1}} \cap$ $\cdots \cap P_{n}^{m_{n}}$, where $\mathbf{m}$ denotes the $n$-tuple $\left(m_{1}, \ldots, m_{n}\right)$ of nonnegative integers $m_{j}$, defines a fat point subscheme $Z=m_{1} p_{1}+\cdots+m_{n} p_{n}$ of $\mathbf{P}^{2}$. (If $m_{1}=\cdots=m_{n}=m$ for some $m$, we will say that $\mathbf{m}$ is uniform and write $(m ; n)$ for $(\mathbf{m} ; n)$. Thus, for example, $I(m ; n)=I((m, \ldots, m) ; n)$, which is the $m$ th symbolic power $I(1 ; n)^{(m)}$ of the ideal $I(1 ; n)$.) Being a homogeneous ideal, $I(\mathbf{m} ; n)$ is a direct sum of its homogeneous components $I(\mathbf{m} ; n)_{t}$ of degree $t$. We can regard $I(\mathbf{m} ; n)_{t}$ as the linear system of forms of degree $t$ with the imposed base point conditions of vanishing at each point $p_{j}$ to order at least $m_{j}$.

These fat point ideals $I(\mathbf{m} ; n)$ have received a lot of attention, with a number of conjectures by various people having been put forward. For example, Nagata ( $[\mathbf{N 1},[\mathbf{N 2}]$ ) has studied the problem of determining $\alpha(\mathbf{m} ; n)$ (i.e., the least degree $t$ such that $\left.I(\mathbf{m} ; n)_{t} \neq 0\right)$ and has conjectured a lower bound. More generally, Harbourne [Ha1, Hirschowitz Hi] and others have put forward conjectures (see Conjecture 4.3 below) for the Hilbert function of $I(\mathbf{m} ; n)$ (i.e., the $k$ vector space dimension $h_{I(\mathbf{m} ; n)}(t)$ of $I(\mathbf{m} ; n)_{t}$ as a function of $\left.t\right)$. If the multiplicities $m_{i}$ all

Received by the editors December 31, 2000 and, in revised form, May 2, 2002.

2000 Mathematics Subject Classification. Primary 13P10, 14C99; Secondary 13D02, 13H15.

Key words and phrases. Ideal generation conjecture, symbolic powers, resolution, fat points, maximal rank.

The first author benefitted from a National Science Foundation grant. 
equal 1, Geramita and Orrechia [GO] conjectured that the minimal free resolution for $I(\mathbf{m} ; n)$ should be $(*)$, given below, and subsequently proved in GGR. However, it is known that $(*)$ fails, in general, even if the multiplicities $m_{i}$ are all equal (see [Ha6], [Ha7] or [FHH]); although, in that case, all known failures involve $n<9$.

In Definition 2.1, we introduce a fairly moderate condition on the multiplicities $m_{i}$ which we call quasiuniformity. Under this condition we show that the conjecture for the Hilbert function of $I(\mathbf{m} ; n)$ takes the following very simple form (subsumed for reasons of technical convenience by our slightly more general version, Conjecture 4.1, which we refer to as the QHC, or Quasiuniform Hilbert Function Conjecture): if $\mathbf{m}$ is quasiuniform, then conjecturally

$$
h_{I(\mathbf{m} ; n)}(t)=\max \left\{0,\left(t^{2}+3 t+2\right) / 2-\sum_{i} m_{i}\left(m_{i}+1\right) / 2\right\} .
$$

Similarly, Conjecture 4.2(b), which we refer to as the QRC, or Quasiuniform Resolution Conjecture, is equivalent to conjecturing that the minimal free resolution in the quasiuniform case maintains the simple behavior proved in [GGR] for general simple points; in particular, if $\mathbf{m}$ is quasiuniform, then the minimal free resolution of $I(\mathbf{m} ; n)$ is, conjecturally,

$(*) \quad 0 \rightarrow R[-a-2]^{a+1-h} \oplus R[-a-1]^{c} \rightarrow R[-a-1]^{b} \oplus R[-a]^{h} \rightarrow I(\mathbf{m} ; n) \rightarrow 0$,

where $a=\alpha(\mathbf{m} ; n), h=h_{I(\mathbf{m} ; n)}(a), b=\max \{0, a+2-2 h\}$ and $c=\max \{0,2 h-a-$ $2\}$. (We recall that $R[-a]^{h}$, for example, denotes the direct sum of $h$ copies of the homogeneous $R$-module $R[-a]$, with the grading defined by $R[-a]_{t}=R_{t-a}$.) We note that the special case of the QRC in which the multiplicities $m_{i}$ are all equal, which we state here as Conjecture 4.2(a) and refer to as the Uniform Resolution Conjecture or URC, was first put forward as Conjecture 6.3 in [Ha6].

The results in Section 3 provide substantial evidence for the URC. Fundamentally, we show that the QHC implies the URC for $I(m ; n)$ for infinitely many $m$ for each $n>9$. For example, if $n$ is an even square bigger than 9, Theorem 3.2 (a) shows for $m$ sufficiently large that $I(m ; n)$ has the conjectured resolution if and only if $\alpha(m ; n)$ has its expected value (this being the value it would have if $h_{I(m ; n)}$ is as conjectured). For such $m$ and $n$, this has the practical benefit that merely by verifying the value of $\alpha(m ; n)$, one obtains the resolution of $I(m ; n)$.

Since in certain cases (see $[\mathrm{AH}]$ and $[\mathrm{E}]$ ) the required Hilbert functions are known, our results also determine resolutions outright, including, for the first time, cases when both $m$ and $n$ can be large, as shown in Corollary 1.1 below. All previous determinations of the resolution of ideals of the form $I(m ; n)$ have assumed either that $m$ is small (such as $m=1[$ GGR] or $m=2$ [Id]) or that $n$ is small $(n \leq 5$ [Cat], or $n \leq 9$ [Ha6]). See Remark 3.6 and Example 4.6] for additional situations where our results give resolutions.

\section{Corollary 1.1.}

(a) For each $m>0$, the QHC and URC both hold for $I(m ; n)$ for infinitely many $n$.

(b) Let $s>1$ and $m \geq(r-2) / 4$ be integers, where $r=2^{s}$ and $n=r^{2}$. If, in addition, the characteristic is 0 , then the $Q H C$ and $Q R C$ both hold for $I(m ; n)$; explicitly, the minimal free resolution of $I(m ; n)$ is

$$
\begin{gathered}
0 \rightarrow R[-a-2]^{a+1-h} \oplus R[-a-1]^{c} \rightarrow R[-a]^{h} \rightarrow I(m ; n) \rightarrow 0, \\
\text { where } a=2^{s} m+2^{s-1}-1, h=\left(a+4^{s-1}+1\right) / 2 \text { and } c=4^{s-1}-1 .
\end{gathered}
$$


See the end of Section 4 for the proof. (Our basic approach uses Lemma 2.4 to convert results on Hilbert functions into results on resolutions. In part (a), we obtain our result on the URC for $m>2$, using the asymptotic result in $\mathrm{AH}$ ] on Hilbert functions; proofs of the URC for $m=1$ and $m=2$ are due to GGR and Id], respectively. Similarly, our result in (b) relies on the determination of the Hilbert function in $\mathrm{E}$ for ideals $I(m ; n)$ with $n$ being a power of 2.)

It may also be of interest to point out that we give one of the best current bounds on the regularity of an ideal of the form $I(m ; n)$; see Remark 2.7. This is used in $\mathrm{HR}$ in verifying the QHC for ideals $I(m ; n)$ for infinitely many $m$ for each square $n>9$. Also, $[\mathrm{HR}$ applies Theorem 3.2 (a) to verify the URC for infinitely many $m$ for each even square $n$, thereby extending Corollary 1.1. (b).

We close the introduction with some remarks of an historical nature. The concerns of this paper are rooted in work by Dubreil $\mathrm{Du}$ ] on numbers of generators of homogeneous ideals and in work by Nagata resolving Hilbert's 14th Problem and posing a still open conjecture ([N1], [N2]) regarding the minimum degree $\alpha$ of a curve with certain assigned multiplicities. Nagata's work can be seen as giving a bound on the Hilbert function of $I(\mathbf{m} ; n)$ for low degrees. Dubreil's work, with additional developments by DGM and Cam, derives from the Hilbert function of $I(\mathbf{m} ; n)$ bounds on numbers of generators of $I(\mathbf{m} ; n)$. More recently, improved bounds have, under certain conditions, been found (see [F1]), and, in various special cases, the minimal free resolution for $I(\mathbf{m} ; n)$ has also been determined: Cat] does so for $n \leq 5$ general points, extended by [F1], [F2] to $n \leq 6$ general points, and now to $n=8$ by $\left[\mathrm{FHH}\right.$; [Ha6] does so for uniform subschemes $m p_{1}+\cdots+m p_{n}$ with $p_{i}$ general and $n \leq 9$; and [Id] does so for $n>9$ general points for subschemes $2 p_{1}+\cdots+2 p_{n}$. However, whereas at least a conjecture has been made for the Hilbert function of $I(\mathbf{m} ; n)$ for arbitrary $\mathbf{m}$ and in this paper we pose and give support for a conjecture for the resolution in the case that $\mathbf{m}$ is quasiuniform, no conjecture for the resolution of $I(\mathbf{m} ; n)$ for arbitrary $\mathbf{m}$ has yet been made.

We would like to thank the referee for carefully reading the paper, and making suggestions to improve the exposition.

\section{Background and Preliminary Lemmas}

We begin by recalling some general facts about resolutions. Because a fat point subscheme of $\mathbf{P}^{2}$ is arithmetically Cohen-Macaulay, the minimal free resolution of $I(\mathbf{m} ; n)$ takes the form $0 \rightarrow F_{1} \rightarrow F_{0} \rightarrow I(\mathbf{m} ; n) \rightarrow 0$, where $F_{0}$ and $F_{1}$ are free graded $R$-modules. Indeed, $F_{0}=\bigoplus_{t} R[-t]^{\nu_{t}}$, where $\nu_{t}$ is the number of homogeneous generators in degree $t$ in $I(\mathbf{m} ; n)$. Alternatively, $\nu_{t}$ is the dimension of the homogeneous component $\left(I(\mathbf{m} ; n) \otimes_{R} k\right)_{t}$, or, equivalently, the dimension of the cokernel of the multiplication map $\mu_{t-1}: I(\mathbf{m} ; n)_{t-1} \otimes R_{1} \rightarrow I(\mathbf{m} ; n)_{t}$. Note that knowing $F_{0}$ and $h_{I(\mathbf{m} ; n)}(t)$ for all $t$ now determines $F_{1}$ up to isomorphism as a graded $R$-module since knowing $F_{0}$ and $h_{I(\mathbf{m} ; n)}$ allows one to determine the Hilbert function of $F_{1}$ and (since $F_{1}$ is free), $F_{1}$ itself. More directly, we have (see [FHH]) $F_{1}=\bigoplus_{i \geq 0} R[-i]^{s_{i}}$ where $\nu_{i}-s_{i}=\Delta^{3} h_{I(\mathbf{m} ; n)}(i)$, where $\Delta$ is the difference operator (hence for any function $f$ we have $\Delta f(i)=f(i)-f(i-1)$, for example).

We will be interested in studying fat point subschemes under certain conditions of uniformity, which we now define. 
Definition 2.1. We will say that $\mathbf{m}$ or more generally $I(\mathbf{m} ; n)$ is uniform if $\mathbf{m}=$ $(m, \ldots, m)$ for some $m \geq 0$ and quasiuniform if $\mathbf{m}=\left(m_{1}, m_{2}, \ldots, m_{n}\right)$ with $n \geq 9$ and $m_{1}=\cdots=m_{9} \geq m_{10} \geq \cdots \geq m_{n} \geq 0$.

Notation 2.2. Given $\mathbf{m}=\left(m_{1}, \ldots, m_{n}\right)$, we will denote $\left(m_{1}+1, m_{2}, \ldots, m_{n}\right)$ by $\mathbf{m}^{+}$and $\left(m_{1}-1, m_{2}, \ldots, m_{n}\right)$ by $\mathbf{m}^{-} ;$likewise, we will write $I(\mathbf{m} ; n)^{+}$for $I\left(\mathbf{m}^{+} ; n\right)$ and, if $m_{1}>0, I(\mathbf{m} ; n)^{-}$for $I\left(\mathbf{m}^{-} ; n\right)$. (If $m_{1}=0$, we will let $I(\mathbf{m} ; n)^{-}=I(\mathbf{m} ; n)$.)

To establish our notation and terminology, consider the space $R_{d}$ of all forms of a given degree $d$. The subspace of those vanishing at a given point of $\mathbf{P}^{2}$ with multiplicity $m$ or more has dimension exactly $\max \{0,((d+1)(d+2)-m(m+1)) / 2\}$. The subspace of those vanishing with multiplicity $m_{i}$ or more at each of $n$ distinct points $p_{i}$ of $\mathbf{P}^{2}$ is just the homogeneous component $I\left(\left(m_{1}, \ldots, m_{n}\right) ; n\right)_{d}$ of the ideal $I(\mathbf{m} ; n)=I\left(\left(m_{1}, \ldots, m_{n}\right) ; n\right)$ generated by all homogeneous forms vanishing with multiplicity $m_{i}$ or more at each point $p_{i}$, and thus has dimension at least $\max \left\{0,\left((d+1)(d+2)-\sum_{i=1}^{n} m_{i}\left(m_{i}+1\right)\right) / 2\right\}$. When the Hilbert function of $I(\mathbf{m} ; n)$ achieves this lower bound in every degree $d$, it is common in the literature to say that $R / I(\mathbf{m} ; n)$ has maximal Hilbert function. Since our focus is on $I(\mathbf{m} ; n)$, we will, in this case, say that the Hilbert function of $I(\mathbf{m} ; n)$ is minimal. Sheafifying $I(\mathbf{m} ; n)$ gives an ideal sheaf $\mathcal{I}$, and we note that $I(\mathbf{m} ; n)$ having minimal Hilbert function is the same thing as $h^{1}\left(\mathbf{P}^{2}, \mathcal{I}(t)\right)$ vanishing for every $t$ for which $h^{0}\left(\mathbf{P}^{2}, \mathcal{I}(t)\right)>0$, where $\mathcal{I}(t)$ denotes the twist $\mathcal{I} \otimes \mathcal{O}_{\mathbf{P}^{2}}(t)$ by $t$ times the class of a line.

Now, although $I(\mathbf{m} ; n)$ does not in general have minimal Hilbert function, the known failures for the Hilbert function to be minimal (which Conjecture 4.3 is formulated to account for) are fairly special, and, in fact, Conjecture 4.3 implies that all quasiuniform $I(\mathbf{m} ; n)$ have minimal Hilbert function (see Remark 4.4). Moreover, CM1 and CM2 show (in characteristic 0) that $I(m ; n)$ has minimal Hilbert function for all $n \geq 10$ as long as $m \leq 12$. Thus, it is reasonable to study resolutions of quasiuniform ideals under assumptions of minimality.

Recall that the Castelnuovo-Mumford regularity of $I(\mathbf{m} ; n)$ is the least degree $t \geq 0$ such that the Hilbert function of $I(\mathbf{m} ; n)$ in degree $t-1$ is equal to $(t(t+1)-$ $\left.\sum_{i=1}^{n} m_{i}\left(m_{i}+1\right)\right) / 2$. Thus, if $I(\mathbf{m} ; n)$ has minimal Hilbert function, it follows that the Castelnuovo-Mumford regularity of $I(\mathbf{m} ; n)$ is at most $\alpha(\mathbf{m} ; n)+1$. This implies (see [DGM] or Lemma 2.9 of [Ha5]) that $\nu_{t}=0$ except for $t=\alpha(\mathbf{m} ; n)$ and possibly $t=\alpha(\mathbf{m} ; n)+1$; i.e., the first syzygy module $F_{0}$ in a minimal free resolution of $I(\mathbf{m} ; n)$ has generators in at most two degrees, $\alpha(\mathbf{m} ; n)$ and $\alpha(\mathbf{m} ; n)+1$. The number $\nu_{\alpha(\mathbf{m} ; n)}$ of generators in degree $\alpha(\mathbf{m} ; n)$ is clearly $h(\mathbf{m} ; n)=h_{I(\mathbf{m} ; n)}(\alpha(\mathbf{m} ; n))$, while if $I(\mathbf{m} ; n)$ has minimal Hilbert function, then the number $\nu_{\alpha(\mathbf{m} ; n)+1}$ of generators in degree $\alpha(\mathbf{m} ; n)+1$ is at least $\max \{0, \alpha(\mathbf{m} ; n)+2-2 h(\mathbf{m} ; n)\}$, since $\alpha(\mathbf{m} ; n)+2-2 h(\mathbf{m} ; n)$ is the difference in dimensions of $I(\mathbf{m} ; n)_{\alpha(\mathbf{m} ; n)+1}$ and $I(\mathbf{m} ; n)_{\alpha(\mathbf{m} ; n)} \otimes R_{1}$. Thus, if $I(\mathbf{m} ; n)$ has minimal Hilbert function, a lower bound on the rank of $F_{0}$ is given by $\max \{h(\mathbf{m} ; n), \alpha(\mathbf{m} ; n)+2-h(\mathbf{m} ; n)\}$. The rank of $F_{0}$ will meet this lower bound if $I(\mathbf{m} ; n)$ has minimal Hilbert function and the multiplication homomorphism $\mu_{\alpha(\mathbf{m} ; n)}: I(\mathbf{m} ; n)_{\alpha(\mathbf{m} ; n)} \otimes R_{1} \rightarrow I(\mathbf{m} ; n)_{1+\alpha(\mathbf{m} ; n)}$ has maximal rank (i.e., is either injective or surjective), in which case all of the multiplication maps $\mu_{t}$ have maximal rank. In this paper we will, under the assumption that the Hilbert function is minimal, study circumstances in which multiplication has maximal rank. Thus, it is convenient to make the following definition: 
Definition 2.3. We will say that maximal rank holds for $I(\mathbf{m} ; n)$ if $I(\mathbf{m} ; n)$ has minimal Hilbert function and $\mu_{\alpha(\mathbf{m} ; n)}$ has maximal rank.

For an ideal $I(\mathbf{m} ; n)$ with minimal Hilbert function, we note that having maximal rank hold is equivalent to the minimal free resolution taking the explicit form $(*)$ given in the introduction. (That $F_{0}$ has the given form is clear. For $F_{1}$, sheafify and take the cohomology of $0 \rightarrow \mathcal{F}_{1}(t) \rightarrow \mathcal{F}_{0}(t) \rightarrow \mathcal{I}(t) \rightarrow 0$ for various twists $t$.)

The following result will be useful. Define $q(\mathbf{m} ; n)$ to be $h_{I\left(\mathbf{m}^{+} ; n\right)}(\alpha(\mathbf{m} ; n))$, and define $l(\mathbf{m} ; n)$ to be $h_{I\left(\mathbf{m}^{-} ; n\right)}(-1+\alpha(\mathbf{m} ; n))$. We will also use $q(m ; n)$ for $q(\mathbf{m} ; n)$ and $l(m ; n)$ for $l(\mathbf{m} ; n)$ in case $\mathbf{m}=\left(m_{1}, \ldots, m_{n}\right)$ when $m=m_{1}=\cdots=m_{n}$.

Lemma 2.4. For each of (a), (b), (c) and (d), if the hypotheses hold for $n$ general points of $\mathbf{P}^{2}$, then so does the conclusion. Assume $m_{i} \geq 0$ for all $i$ and $m_{1}>0$ in $\mathbf{m}=\left(m_{1}, \ldots, m_{n}\right)$.

(a) If $I(\mathbf{m} ; n)$ has minimal Hilbert function, $q(\mathbf{m} ; n)=0$ and $l(\mathbf{m} ; n)=0$, then $\mu: I(\mathbf{m} ; n)_{\alpha(\mathbf{m} ; n)} \otimes R_{1} \rightarrow I(\mathbf{m} ; n)_{1+\alpha(\mathbf{m} ; n)}$ is injective and hence maximal rank holds for $I(\mathbf{m} ; n)$.

(b) If $I(\mathbf{m} ; n)$ has minimal Hilbert function, $\mathbf{m}=\left(m_{1}, \ldots, m_{n}\right)$ has $m_{1}=m_{2}$, and $q(\mathbf{m} ; n)=0$, then $\mu$ is injective and so maximal rank holds for $I(\mathbf{m} ; n)$.

(c) If $I(\mathbf{m} ; n), I(\mathbf{m} ; n)^{+}$and $I(\mathbf{m} ; n)^{-}$all have minimal Hilbert functions, $\mathbf{m}=$ $\left(m_{1}, \ldots, m_{n}\right)$ has $m_{1}=m_{2}$, and $l(\mathbf{m} ; n)>0$, then $\mu$ is surjective and so maximal rank holds for $I(\mathbf{m} ; n)$.

(d) More generally, if $I(\mathbf{m} ; n), I(\mathbf{m} ; n)^{+}$and $I(\mathbf{m} ; n)^{-}$all have minimal Hilbert functions and if both $l(\mathbf{m} ; n)$ and $q(\mathbf{m} ; n)$ are positive, then $\mu$ is surjective and so maximal rank holds for $I(\mathbf{m} ; n)$.

Proof. (a) Keeping in mind our comment about regularity (preceding Definition 2.3), this follows from Lemma 4.1 of [Ha6].

(b) Let $f$ be the linear form vanishing on the line through the points $p_{1}$ and $p_{2}$. Then multiplication by $f$ gives an injection $I\left(-1+m_{1}, m_{2}, m_{3}, \ldots, m_{n} ; n\right)_{-1+\alpha(\mathbf{m} ; n)}$ $\rightarrow I\left(m_{1}, 1+m_{2}, m_{3}, \ldots, m_{n} ; n\right)_{\alpha(\mathbf{m} ; n)}$, and since the points $p_{1}, \ldots, p_{n}$ are general, we know $I\left(m_{1}, 1+m_{2}, m_{3}, \ldots, m_{n} ; n\right)_{\alpha(\mathbf{m} ; n)}$ and $I\left(1+m_{1}, m_{2}, \ldots, m_{n} ; n\right)_{\alpha(\mathbf{m} ; n)}$ have the same dimension; hence $l(\mathbf{m} ; n) \leq q(\mathbf{m} ; n)$. Therefore, if $q(\mathbf{m} ; n)=0$, then maximal rank holds for $I(\mathbf{m} ; n)$ by $(\mathrm{a})$.

(c) As in (b), we have $l(\mathbf{m} ; n) \leq q(\mathbf{m} ; n)$; so this follows from (d), which follows from Lemma 4.2 of [Ha6] by applying the hypotheses of minimality for the Hilbert function.

Our underlying approach uses geometrical arguments, based on the blow-up $X$ of $\mathbf{P}^{2}$ at the $n$ points $p_{1}, \ldots, p_{n}$. Results in this geometrical setting directly translate back to the algebraic setting to which we have, up to now, mostly confined ourselves, and we refer the reader to, for example, Section 3 of [Ha6], for the dictionary to do so. Briefly, if $X \rightarrow \mathbf{P}^{2}$ is the blow-up of the points $p_{1}, \ldots, p_{n}$, we denote by $L$ the total transform to $X$ of a line in $\mathbf{P}^{2}$, and by $E_{i}$ the exceptional locus of the blow-up of $p_{i}$. Then the divisor classes $[L],\left[E_{1}\right], \ldots,\left[E_{n}\right]$ give a basis of the divisor class group of $X$. We will denote the divisor $t L-m\left(E_{1}+\cdots+E_{n}\right)$ by $F_{t, m}$, and the corresponding line bundle $\mathcal{O}_{X}\left(F_{t, m}\right)$ by $\mathcal{F}_{t, m}$. Note that $[L]=\left[F_{1,0}\right]$ and $\left[F_{t, l}\right]+\left[F_{s, m}\right]=\left[F_{t+s, l+m}\right]$.

In the case that $n$ is a square, our treatment of $I(m ; n)$ will depend on whether $n$ is even or whether it is odd. We accumulate some of the geometrical results we will need. 
Lemma 2.5. Let $t$ and $m \geq 0$ be integers and let $X$ be the blow-up of $\mathbf{P}^{2}$ at $n=r^{2}$ general points (the conditions of generality depend on both $n$ and $m$ ).

(i) Let $r$ be even.

(a) Then $h^{1}\left(X, \mathcal{F}_{t, m}\right)=0$ and $h^{0}\left(X, \mathcal{F}_{t, m}\right)>0$ for all $t \geq r m+(r-2) / 2$.

(b) If $0 \leq t<r m+(r-2) / 2$ and $m \geq(r-2) / 4$, then $h^{0}\left(X, \mathcal{F}_{t, m}\right)-$ $h^{1}\left(X, \mathcal{F}_{t, m}\right) \leq 0$, with $h^{1}\left(X, \mathcal{F}_{t, m}\right)>0$ if $m>(r-2) / 4$.

(ii) Let $r \geq 3$ be odd.

(a) Then $h^{1}\left(X, \mathcal{F}_{t, m}\right)=0$ and $h^{0}\left(X, \mathcal{F}_{t, m}\right)>0$ for all $t \geq r m+(r-3) / 2$.

(b) If $0 \leq t<r m+(r-3) / 2$ and $m \geq(r-1)(r-3) /(8 r)$, then $h^{0}\left(X, \mathcal{F}_{t, m}\right)-h^{1}\left(X, \mathcal{F}_{t, m}\right) \leq 0$, with $h^{1}\left(X, \mathcal{F}_{t, m}\right)>0$ if $m>(r-$ $1)(r-3) /(8 r)$.

Proof. Note for $t \geq 0$, that $h^{2}\left(X, \mathcal{F}_{t, m}\right)=h^{0}\left(X, \mathcal{F}_{-3-t,-1-m}\right)$ by duality, and $h^{0}\left(X, \mathcal{F}_{-3-t,-1-m}\right)$ vanishes, since $-3-t<0$. Thus, for both (i)(b) and (ii)(b) we have $h^{0}\left(X, \mathcal{F}_{t, m}\right)-h^{1}\left(X, \mathcal{F}_{t, m}\right)=\left(F_{t, m}^{2}-K_{X} \cdot F_{t, m}+2\right) / 2$ by Riemann-Roch. Now $F_{t, m}^{2}-K_{X} \cdot F_{t, m}+2=(t+2)(t+1)-r^{2} m(m+1)$ is an increasing function of $t$ for $t \geq 0$; so to prove (i)(b) and (ii)(b) it is enough to take $t=r m+(r-2) / 2-1$ when $r$ is even and $t=r m+(r-3) / 2-1$ when $r$ is odd. For $t=r m+(r-2) / 2-1$, $(t+2)(t+1)-r^{2} m(m+1)$ becomes $(r / 4)(r-4 m-2)$, which is 0 for $m=(r-2) / 4$ and negative for $m>(r-2) / 4$. This proves (i)(b). For $t=r m+(r-3) / 2-1$, $(t+2)(t+1)-r^{2} m(m+1)$ becomes $-2 r m+(r-3)(r-1) / 4$, which is 0 for $m=(r-3)(r-1) /(8 r)$ and negative for $m \geq(r-3)(r-1) /(8 r)$. This proves (ii) (b).

To prove both parts (a), consider a specialization of the $r^{2}$ points to the case of general points $p_{i}$ on a smooth plane curve $C^{\prime}$ of degree $r$, and let $C$ be the proper transform to $X$ of $C^{\prime}$. In the case that $r$ is even (so $t \geq r m+(r-2) / 2$ ), the restriction $\mathcal{F}_{t, m} \otimes \mathcal{O}_{C}$ to $C$ has degree at least $(r-2) / 2+g$, where $g$ is the genus of $C$. But $\mathcal{F}_{t, m} \otimes \mathcal{O}_{C}$ is a general bundle of its degree (the points $p_{i}$ being general points on $C^{\prime}$ ); so $\mathcal{F}_{t, m}^{-1} \otimes \mathcal{K}_{C}$ is a general bundle of degree at most $g-r / 2-1$, and thus has no nontrivial global sections ( $\operatorname{since} \operatorname{Pic}^{0}(C)$ has dimension $g$, there are more line bundles than there are effective divisors for any given degree less than $g$ ). Thus, $h^{1}\left(C, \mathcal{F}_{t, m} \otimes \mathcal{O}_{C}\right)=0$ by duality. Since (i)(a) is true for $m=0$, our result follows for all $m \geq 0$ by induction by taking the cohomology of the sequence

$$
0 \rightarrow \mathcal{F}_{t-r, m-1} \rightarrow \mathcal{F}_{t, m} \rightarrow \mathcal{F}_{t, m} \otimes \mathcal{O}_{C} \rightarrow 0
$$

obtained by restriction. The result for general points of $\mathbf{P}^{2}$ (rather than general points of $C^{\prime}$ ) now follows by semicontinuity. Case (ii)(a), that $r$ is odd, is similar, except now $t \geq r m+(r-3) / 2$ and $\mathcal{F}_{t, m} \otimes \mathcal{O}_{C}$ has degree at least $g-1$.

Remark 2.6. To justify use of semicontinuity in the preceding proof, we can appeal to flat families, using results of [Ha2]. Alternatively, consider any $n$ nonnegative multiplicities $m_{1}, \ldots, m_{n}$ and any $n$ distinct points $p_{1}, \ldots, p_{n} \in \mathbf{P}^{2}$, with their corresponding ideals $P_{i}$. We may assume that none of the ideals $P_{i} \subset R=k[x, y, z]$ contain $z$. Let $I=\bigcap_{i=1}^{n} P_{i}^{m_{i}}$ and let $V_{i}$ be the vector space span in $k[x, y]$ of the monomials of degree less than $m_{i}$. For each degree $t$ and each point $p_{i}$, we have a linear map $\lambda_{t i}: R_{t} \rightarrow V_{i}$, in which a homogeneous polynomial $f(x, y, z)$ of degree $t$ is first sent to $f\left(x+x_{i}, y+y_{i}, 1\right)$, where $\left(x_{i}, y_{i}\right)$ are affine coordinates for $p_{i}$ (taking $z=0$ to be the line at infinity), and then $f\left(x+x_{i}, y+y_{i}, 1\right)$ is truncated to drop all terms of degree $m_{i}$ or more. With $V$ taken to be $V_{1} \times \cdots \times V_{n}$, define $\Lambda_{t}: R_{t} \rightarrow V$ to 
be the map $\lambda_{t 1} \times \cdots \times \lambda_{t n}$. Thus, the kernel of $\Lambda_{t}$ is $I_{t}$, and the entries for the matrix defining $\Lambda_{t}$ in terms of bases of monomials are polynomials in the coordinates of the points $p_{i}$. If $X$ is the blow-up of $\mathbf{P}^{2}$ at the points $p_{i}$, and if we set $\mathcal{F}=\mathcal{O}_{X}(F)$ where $F=t L-m_{1} E_{1}-\cdots-m_{n} E_{n}$, then $h^{0}(X, \mathcal{F})=\operatorname{dim} I_{t}$ and by RiemannRoch, $h^{0}(X, \mathcal{F})-h^{1}(X, \mathcal{F})=\operatorname{dim} R_{t}-\operatorname{dim} V$. Since $\operatorname{dim} I_{t}=\operatorname{dim} R_{t}-l$ where $l$ is the rank of $\Lambda_{t}$, we see that $h^{1}(X, \mathcal{F})=\operatorname{dim} V-l$. But for any given $l, \Lambda_{t}$ having rank at least $l$ is an open condition on the set of all $n$-tuples $\left(p_{1}, \ldots, p_{n}\right)$ of distinct points with none at infinity, and hence $h^{1}(X, \mathcal{F})$ is also semicontinuous.

Remark 2.7. Note that Lemma 2.5 determines the regularity of $I(m ; n)$ for each square $n>1$ for all $m$ sufficiently large, and it implies more generally that the regularity of $I(m ; n)$ for any $n \geq 9$ general points and any $m$ is at most $1+$ $\lceil\sqrt{n}\rceil m+\lceil(\lceil\sqrt{n}\rceil-3) / 2\rceil$. This is often the best bound on the regularity currently known. For example, in [Gi2] and [Hi] a bound of approximately $m \sqrt{2 n}$ is given, while $[\mathbf{X}]$ obtains a bound of $\lfloor(m+1) \sqrt{10 n / 9}-1\rfloor$. More recently, $[\mathbb{R}]$ gives a bound of $\lfloor(m+1)(\sqrt{n+1.9}+\pi / 8)\rfloor$, but for $m$ sufficiently large our bound is better for approximately $40 \%$ of the values of $n$ between any two consecutive squares, with our bound being sharp when $n$ is square.

Our proof of Theorem [3.2(a) involves examining certain specializations of the $r^{2}$ points. The basic idea for the specialization that we will use comes from GGR. However, in GGR the specialization was used to draw conclusions about general points of multiplicity 1 . In our situation, we first specialize the points to a curve so that we can do an induction to reduce points of multiplicity $m$ to points of multiplicity 1 . But these points of multiplicity 1 are now on the curve; so we need to modify the specialization of GGR to work for points that have already been specialized to the curve. We then can draw conclusions about general points from our conclusions about the points on the curve.

In our modification of [GGR, the points will lie on a curve of degree $r$, where $r$ is even. Choose coordinates $x, y$ and $z$ on $\mathbf{P}^{2}$. Our specialization is easiest to specify as a subset of the affine plane $\mathbf{A}^{2}=\mathbf{P}^{2}-\{z=0\}$, rather than of $\mathbf{P}^{2}$. Regard $\mathbf{A}^{2}$ as $k^{2}$, where $k$ is the ground field. Equations of vertical lines now have the form $x-c$, for $c \in k$, and equations of horizontal lines have the form $y-c$, for $c \in k$. Consider $r$ distinct vertical lines $V_{1}, \ldots, V_{r}$ in $k^{2}$ and $s=3 r / 2-1$ distinct horizontal lines $H_{1}, \ldots, H_{s}$. Let $p_{i j}$, for each $1 \leq i \leq r$ and $1 \leq j \leq s$, be the point of intersection of $V_{i}$ with $H_{j}$. We choose our $r^{2}$ points in blocks from among these $r s$ points $p_{i j}$. The first block is $B_{1}=\left\{p_{i j}: 1 \leq i \leq 2,1 \leq j \leq s\right\}$, the second is $B_{2}=\left\{p_{i j}: 3 \leq i \leq 4,1 \leq j \leq s-2\right\}$, etc., and the last block is $B_{r / 2}=\left\{p_{i j}: r-1 \leq i \leq r, 1 \leq j \leq s-(r-2)\right\}$. Note that all together, the union of these $r / 2$ blocks contains $2 s+\cdots+2(s-(r-2))=2(s r / 2-(2+4+\cdots+(r-2)))=$ $s r-4(1+\cdots+(r / 2-1))=(3 r / 2-1) r-4(r / 2-1)(r / 2) / 2=r^{2}$ points. It will be convenient to denote $r m+(r-2) / 2$ by $t_{m}$ and the natural multiplication map $H^{0}\left(X, \mathcal{F}_{t_{m}, m}\right) \otimes H^{0}\left(X, \mathcal{F}_{1,0}\right) \rightarrow H^{0}\left(X, \mathcal{F}_{t_{m}+1, m}\right)$ by $\mu_{t_{m}}$.

Proposition 2.8. Let $r$ be even. With respect to the configuration $B_{1} \cup \cdots \cup B_{r / 2}$ of $r^{2}$ points of $\mathbf{P}^{2}$ specified above, $\mu_{t_{1}}$ is surjective and $h^{1}\left(X, \mathcal{F}_{t_{1}, 1}\right)=0$.

Proof. Apply the method and results of GGR. Let $I \subset R$ be the homogeneous ideal of the $r^{2}$ points in the homogeneous coordinate ring $R=k[x, y, z]$ of $\mathbf{P}^{2}$, with coordinates as specified in the paragraph preceding the statement of the proposition. Since $z$ does not vanish on any of the $r^{2}$ points, the image of $z$ in the quotient $R / I$ 
is not a zero divisor. Hence, as discussed in GGR, $J=I+(z) /(z)$ has the same minimal number of homogeneous generators in every degree as does $I$, but $J$ is a monomial ideal which, by the discussion in GGR], is easy to handle explicitly. The result in our case is that there are no generators in degrees greater than $3 r / 2-1$; hence the same is true for $I$, which shows that $\mu_{t_{1}}$ is surjective, as required. To see that $h^{1}\left(X, \mathcal{F}_{t_{1}, 1}\right)=0$, it is enough to check that the points impose independent conditions on forms of degree $t_{1}$; i.e., that $h^{0}\left(X, \mathcal{F}_{t_{1}, 1}\right)=\left(t_{1}+2\right)\left(t_{1}+1\right) / 2-r^{2}$. But the fact that $J=I+(z) /(z)$ has the same number of generators in every degree as does $I$ means that $I$ has one generator in degree $r$ and $r / 2$ in degree $t_{1}=3 r / 2-1$. Thus, the dimension of $I$ in degree $t_{1}$ is $(r / 2)+((r / 2)(r / 2+1) / 2)$, which is indeed $\left(t_{1}+2\right)\left(t_{1}+1\right) / 2-r^{2}$.

Proposition 2.9. Let $r \geq 0$ be even with $m \geq 0$. Then for general points $p_{1}, \ldots$, $p_{r^{2}}$ of a general smooth plane curve $C^{\prime}$ of degree $r$, taking $X$ to be the blow-up of $\mathbf{P}^{2}$ at $p_{1}, \ldots, p_{r^{2}}$, the map $\mu_{t_{m}}$ is surjective and $h^{1}\left(X, \mathcal{F}_{t_{m}, m}\right)=0$ for all $m \geq 0$.

Proof. First let $C^{\prime}$ be any smooth plane curve of degree $r$ and let $C$ be the proper transform of $C^{\prime}$ to $X$. As in the proof of Lemma 2.5 we have the exact sequence $0 \rightarrow \mathcal{F}_{t_{m}-r, m-1} \rightarrow \mathcal{F}_{t_{m}, m} \rightarrow \mathcal{F}_{t_{m}, m} \otimes \mathcal{O}_{C} \rightarrow 0$, and $h^{1}\left(X, \mathcal{F}_{t_{m}, m}\right)=0$ for all $m \geq 0$. The exact sequence leads by the snake lemma (see [Mu] or [Ha5]) to an exact sequence,

$$
\operatorname{cok}\left(\mu_{t_{m}-r}\right) \rightarrow \operatorname{cok}\left(\mu_{t_{m}}\right) \rightarrow \operatorname{cok}\left(\mu_{C, t_{m}}\right) \rightarrow 0
$$

where $\mu_{C, t_{m}}$ is the map $H^{0}\left(C, \mathcal{F}_{t_{m}, m} \otimes \mathcal{O}_{C}\right) \otimes H^{0}\left(X, \mathcal{F}_{1,0}\right) \rightarrow H^{0}\left(X, \mathcal{F}_{t_{m}+1, m} \otimes \mathcal{O}_{C}\right)$. But $\mathcal{F}_{t_{m}, m} \otimes \mathcal{O}_{C}$ is a general bundle of degree $r(r-2) / 2$, regardless of $m$; so if $\operatorname{cok}\left(\mu_{C, t_{1}}\right)=0$, then cok $\left(\mu_{C, t_{m}}\right)=0$ for all $m \geq 1$, and cok $\left(\mu_{C, t_{1}}\right)=0$ follows from $(* *)$ if we show that $\operatorname{cok}\left(\mu_{t_{1}}\right)=0$. But $\operatorname{cok}\left(\mu_{t_{1}}\right)=0$ and $h^{1}\left(X, \mathcal{F}_{t_{1}, 1}\right)=0$ for the configuration of points given in Proposition 2.8] and these points are points of a plane curve $C^{\prime}$ of degree $r$ (the union of $r$ lines); so by semicontinuity (see Remark 2.10) cok $\left(\mu_{t_{1}}\right)=0$ holds for general points of a general curve $C^{\prime}$ of degree $r$.

Finally, induction using $(* *)$ gives $\operatorname{cok}\left(\mu_{t_{m}}\right)=0$ for all $m \geq 0$. (Note that $\mu_{t_{m}-r}=\mu_{t_{m-1}}$ and so $\mu_{t_{m}-r m}=\mu_{t_{0}}$, and cok $\left(\mu_{t_{m}-r m}\right)=0$ since $\mathcal{F}_{t_{m}-r m, 0}=$ $\mathcal{F}_{t_{0}, 0}$ can be regarded as $\mathcal{O}_{\mathbf{P}^{2}}(((r-2) / 2) L)$ on $\mathbf{P}^{2}$, where the result is obvious.)

Remark 2.10. We now explain why the requirements that cok $\left(\mu_{t_{1}}\right)=0$ and $h^{1}\left(X, \mathcal{F}_{t_{1}, 1}\right)=0$ together impose an open condition on $r^{2}$-tuples of points $\left(p_{1}, \ldots\right.$, $\left.p_{r^{2}}\right)$. More generally, let $I$ and $\mathcal{F}$ be as in Remark 2.6. As there, we have the map $\Lambda_{t}: R_{t} \rightarrow V$. Then $I_{t} \otimes R_{1}$ is the kernel of $\Lambda_{t} \otimes \mathrm{id}_{R_{1}}: R_{t} \otimes R_{1} \rightarrow V \otimes R_{1}$ and the kernel of $\mu_{t}: I_{t} \otimes R_{1} \rightarrow I_{t+1}$ is also the kernel of $\gamma: R_{t} \otimes R_{1} \rightarrow\left(V \otimes R_{1}\right) \oplus R_{t+1}$, where $\gamma$ is $\left(\Lambda_{t} \otimes \mathrm{id}_{R_{1}}\right) \oplus \mu_{t}^{\prime}$, and where $\mu_{t}: I_{t} \otimes R_{1} \rightarrow I_{t+1}$ and $\mu_{t}^{\prime}: R_{t} \otimes R_{1} \rightarrow R_{t+1}$ are given by multiplication. (This is consistent with our usage above, since sections of line bundles on blow-ups of $\mathbf{P}^{2}$ can be identified with subspaces of $R_{t}$ for appropriate $t$.) Since, as in Remark 2.6, $\gamma$ can be given by a matrix whose entries are polynomials in the coordinates of the points $p_{i}$, the rank of $\gamma$ and hence the dimension of ker $\left(\mu_{t}\right)$ is semicontinuous. But dim cok $\left(\mu_{t}\right)=h^{0}(X, \mathcal{F}(L))-3 h^{0}(X, \mathcal{F})+\operatorname{dim} \operatorname{ker}\left(\mu_{t}\right)$, which is $\operatorname{dim} \operatorname{cok}\left(\mu_{t}\right)=t+F \cdot K_{X}-F^{2}+\operatorname{dim} \operatorname{ker}\left(\mu_{t}\right)$ if $h^{1}(X, \mathcal{F})=0$, and since $h^{1}(X, \mathcal{F})$ is also semicontinuous, it follows that it is an open condition to require that both cok $\left(\mu_{t}\right)$ and $h^{1}(X, \mathcal{F})$ vanish. 
We will say that an effective, reduced, irreducible divisor $A$ on a surface $X$ is a $(-1)$-curve if $A^{2}=A \cdot K_{X}=-1$. For later use, we now show how quasiuniformity gives us control over intersections with $(-1)$-curves.

Lemma 2.11. Let $X$ be a blow-up of $\mathbf{P}^{2}$ at $n$ general points, and let $D_{t}=t L-$ $m_{1} E_{1}-\cdots-m_{n} E_{n}, D_{t}^{+}=t L-\left(m_{1}+1\right) E_{1}-m_{2} E_{2}-\cdots-m_{n} E_{n}$ and $D_{t}^{-}=$ $t L-\left(m_{1}-1\right) E_{1}-m_{2} E_{2}-\cdots-m_{n} E_{n}$, where, as above, $L, E_{1}, \ldots, E_{n}$ correspond to the usual basis of the divisor class group of $X$. If $\left(m_{1}, \ldots, m_{n}\right)$ is quasiuniform with $m_{1}>0$ and $\left|D_{t}\right|$ is nonempty, then $D_{t} \cdot A \geq 0$ for any $(-1)$-curve $A$ (and likewise with $D_{t}$ replaced by either $D_{t}^{+}$or $D_{t}^{-}$).

Proof. By quasiuniformity we know that $m_{1}=\cdots=m_{9}$ (we will denote this common multiplicity by $m$ ), and $m \geq m_{10} \geq \cdots \geq m_{n}$. But for $C=3 L-E_{1}-\cdots-$ $E_{9}$, we have $C \cdot D \geq 0$ for any effective divisor $D$, since there is always an irreducible cubic through 9 general points. If $\left|D_{t}\right|$ is nonempty, it follows that $C \cdot D_{t} \geq 0$; hence $t \geq 3 m$ and so $D_{t}=-m K_{X}+(t-3 m) L+\left(m-m_{10}\right) E_{10}+\cdots+\left(m-m_{n}\right) E_{n}$. If $A=E_{i}$ for some $i$, then $D_{t} \cdot A \geq 0$, since $E_{i} \cdot D_{t} \geq 0$ for all $i$ by quasiuniformity. If $A$ is not $E_{i}$ for any $i$, then again $D_{t} \cdot A \geq 0$, since $A \cdot D_{t} \geq A \cdot\left(-m K_{X}\right)=m \geq 0$.

Next assume that $\left|D_{t}^{+}\right|$is nonempty. We now have $C \cdot D_{t}^{+} \geq 0$; so $3 t \geq 9 m+1$ or $t \geq 3 m+1$ and then $D_{t}^{+}=-m K_{X}+(t-3 m) L+\left(L-E_{1}\right)+\left(m-m_{10}\right) E_{10}+$ $\cdots+\left(m-m_{n}\right) E_{n}$ and the argument now proceeds as before. Finally, assume that $\left|D_{t}^{-}\right|$is nonempty. Then $3 t \geq 9 m-1$; so $t \geq 3 m$ and then $D_{t}^{-}=-m K_{X}+(t-$ $3 m) L+E_{1}+\left(m-m_{10}\right) E_{10}+\cdots+\left(m-m_{n}\right) E_{n}$ and again we obtain the result.

\section{Main Results}

We obtain results separately for even squares, odd squares and nonsquares. We briefly discuss why our results differ depending on whether $n$ is an even square, an odd square, or not a square. As discussed above, if $I(m ; n)$ has minimal Hilbert function, the problem is to show that $\mu_{\alpha(m ; n)}$ has maximal rank. In those cases when $\mu_{\alpha(m ; n)}$ is injective, we can try to verify injectivity by applying the criterion $q(m ; n)=0=l(m ; n)$ of Lemma [2.4(a). Assuming minimality, when $n>9$ is a nonsquare, it turns out that $q(m ; n)=0=l(m ; n)$ for infinitely many $m$ (see Proposition [3.1), and if $n>9$ is an odd square, it again turns out that $q(m ; n)=$ $0=l(m ; n)$, but now for all sufficiently large $m$ (see Theorem 3.2(b)). This approach was already employed in [Ha6], but only for certain special values of $n$; thus, we are now extending this to all $n$ that are not even squares. When $n>9$ is an even square, it turns out that $\mu_{\alpha(m ; n)}$ fails to be injective for all sufficiently large $m$. Assuming minimality, we instead verify that $\mu_{\alpha(m ; n)}$ is surjective for $m$ sufficiently large. To do this, we use a specialization argument applying Proposition 2.9.

Proposition 3.1. Let $n>9$ be a nonsquare. Then there are infinitely many $m$ such that if $I(m ; n)$ and $I(m ; n)^{+}$have minimal Hilbert function for $n$ general points of $\mathbf{P}^{2}$, then maximal rank holds for $I(m ; n)$.

Proof. We will use a criterion developed in the proof of Corollary 5.9 of [Ha6], which we briefly recall. By Lemma 2.4, if $I(m ; n)$ has minimal Hilbert function and $q(m ; n)=0$, then maximal rank holds for $I(m ; n)$. If $I(m ; n)$ has minimal Hilbert function, $\alpha(m ; n)$ is the solution $x$ to the pair of inequalities $(x+1)(x+2)-n(m+$ $1) m>0$ and $(x+1) x-n(m+1) m \leq 0$. If, in addition, $I(m ; n)^{+}$has minimal Hilbert function, then $q(m ; n)=0$ if and only if also $(x+1)(x+2)-n(m+1) m-2(m+1) \leq 0$. 
But $(x+1)(x+2)-n(m+1) m>0$ implies that $x \geq m$, and since adding $2(x-m)$ to $(x+1) x-n(m+1) m$ gives $(x+1)(x+2)-n(m+1) m-2(m+1)$, we see, assuming $I(m ; n)$ and $I(m ; n)^{+}$have minimal Hilbert function, that $q(m ; n)=0$ if $(x+1)(x+2)-n(m+1) m>0$ and $(x+1)(x+2)-n(m+1) m-2(m+1) \leq 0$ have a simultaneous positive integer solution $x$. Let $0<\epsilon<1$; by easy but tedious arithmetic, one can check that each real $x$ in the interval $[\sqrt{n} m+(\sqrt{n}-$ $3) / 2, \sqrt{n} m+(\sqrt{n}-3) / 2+\epsilon / \sqrt{n}]$ is, for $m$ sufficiently large (how large depending on $n$ and $\epsilon$ ), a solution to our pair of inequalities. Thus this interval containing an integer is a criterion for $q(m ; n)$ to vanish. But by simplifying, there being an integer $x$ in this interval is equivalent to there being an integer $\eta=x+1$ satisfying $0<(2 \eta+1) /(2 m+1)-\sqrt{n} \leq 2 \epsilon /((2 m+1) \sqrt{n})$.

So now we consider $0<(2 \eta+1) /(2 m+1)-\sqrt{n} \leq 2 \epsilon /((2 m+1) \sqrt{n})$. Since $n$ is not a square, we can write $n=a^{2}+b$ with $0<b \leq 2 a$, by taking $a=[\sqrt{n}]$ to be the integer part of $\sqrt{n}$. We now show that there are infinitely many pairs of odd integers $p, q$ such that $0<p / q-\sqrt{a^{2}+b} \leq 2 \epsilon /\left(q \sqrt{a^{2}+b}\right)$, thus completing the proof.

Let $f$ and $g$ be positive odd integers such that $f^{2}-\left(a^{2}+b\right) g^{2}$ is positive. As is well known, Pell's equation, $z^{2}-\left(a^{2}+b\right) y^{2}=1$, has a solution $z=c, y=d$ in positive integers, and we obtain additional solutions $z=u^{\prime}, y=v^{\prime}$ from $u^{\prime}+v^{\prime} \sqrt{a^{2}+b}=$ $\left(c+d \sqrt{a^{2}+b}\right)^{t}$. Moreover, whenever $t$ is even, it is easy to check that $u^{\prime}$ is odd and $v^{\prime}$ is even. Now, taking $u+v \sqrt{a^{2}+b}=\left(u^{\prime}+v^{\prime} \sqrt{a^{2}+b}\right)\left(f+g \sqrt{a^{2}+b}\right)$, we obtain a solution $z=u, y=v$ to $z^{2}-\left(a^{2}+b\right) y^{2}=f^{2}-\left(a^{2}+b\right) g^{2}$ with $u$ and $v$ both odd. It follows that there are infinitely many such solutions. Moreover, $u / v-\sqrt{a^{2}+b}=$ $\left(f^{2}-\left(a^{2}+b\right) g^{2}\right) /\left(v^{2}\left(u / v+\sqrt{a^{2}+b}\right)\right)<\left(f^{2}-\left(a^{2}+b\right) g^{2}\right) /\left(v^{2} \sqrt{a^{2}+b}\right)$, which is clearly less than or equal to $2 \epsilon /\left(v \sqrt{a^{2}+b}\right)$ for $v$ sufficiently large.

Corollary 5.8 of [Ha6] shows that Proposition 3.1]also holds for odd squares $n>9$. Theorem 3.2 (b) strengthens this result for odd squares. In addition, Theorem 3.2 (a) obtains an even stronger result for even squares. It is, by Lemma 2.5](i), a precise formulation of the fact that for each sufficiently large $m$, if $I(m ; n)$ has minimal Hilbert function for $n$ general points when $n$ is an even square bigger than 9, then maximal rank holds for $I(m ; n)$.

Theorem 3.2. Consider $n=r^{2}$ general points of $\mathbf{P}^{2}$, where $r \geq 3$.

(a) Assume $r$ is even and $m \geq(r-2) / 4$. Then $I(m ; n)$ has minimal Hilbert function if and only if $\alpha(m ; n)=r m+r / 2-1$, in which case maximal rank holds for $I(m ; n)$.

(b) Assume $r$ is odd and $m \geq\left(r^{2}-9\right) / 8$. Then, for $n$ general points of $\mathbf{P}^{2}$, maximal rank holds for $I(m ; n)$ if $I(m ; n)$ and $I(m ; n)^{+}$have minimal Hilbert function.

Proof. (a) First, $\alpha(m ; n)=r m+r / 2-1$ if and only if $I(m ; n)_{t}=0$ for $t<$ $r m+r / 2-1$, since $h^{0}\left(X, \mathcal{F}_{t, m}\right)>0$ for $t \geq r m+r / 2-1$ by Lemma 2.5)(i)(a). Again by Lemma [2.5(i)(a), we have $h^{1}\left(X, \mathcal{F}_{t, m}\right)=0$ for $t \geq r m+r / 2-1$ and, if $m \geq(r-2) / 4, h^{0}\left(X, \mathcal{F}_{t, m}\right) \leq h^{1}\left(X, \mathcal{F}_{t, m}\right)$ for $t<r m+r / 2-1$; so $I(m ; n)$ has minimal Hilbert function if and only if $I(m ; n)_{t}=0$ for $t<r m+r / 2-1$.

Next, given that $I(m ; n)_{t}=0$ for $t<r m+r / 2-1$, there are no homogeneous generators for $I(m ; n)$ in degrees less than $r m+r / 2-1$ and there are $h_{I(m ; n)}(\alpha(m ; n))$ generators in degree $r m+r / 2-1$. Clearly, then, maximal rank holds for $I(m ; n)$ if 
$F_{0}$ has rank $h_{I(m ; n)}(\alpha(m ; n))$; so it is enough to prove that these $h_{I(m ; n)}(\alpha(m ; n))$ elements of degree $r m+r / 2-1$ generate $I(m ; n)$.

By Lemma 2.5)(i), the Castelnuovo-Mumford regularity of $I(m ; n)$ is at most $\alpha(m ; n)+1$; so no generators need be taken in degrees greater than $r m+r / 2$ (see [DGM] or Lemma 2.9 of [Ha5]). Hence, we now need only to show that no generators need be taken in degree $r m+r / 2$; i.e., that $\mu_{t_{m}}: H^{0}\left(X, \mathcal{F}_{t_{m}, m}\right) \otimes H^{0}\left(X, \mathcal{F}_{1,0}\right) \rightarrow$ $H^{0}\left(X, \mathcal{F}_{t_{m}+1, m}\right)$ is surjective, where, as in Proposition 2.9, $t_{m}=r m+r / 2-1$. But this follows by Proposition 2.9 and semicontinuity (cf. Remark 2.10).

(b) (We note that when $n=r^{2}>9$ is an odd square, [Ha6 noted but did not explicitly show that maximal rank holds for $I\left(m ; r^{2}\right)$ for all but finitely many $m$ for which $I\left(m ; r^{2}\right)$ and $I\left(m ; r^{2}\right)^{+}$have minimal Hilbert function.) By Lemma 2.4(b), if $I\left(m ; r^{2}\right)$ has minimal Hilbert function and $q\left(m ; r^{2}\right)=0$, then maximal rank holds for $I\left(m ; r^{2}\right)$. But Lemma 2.5(ii) implies that $\alpha\left(m ; r^{2}\right)=r m+(r-3) / 2$ for $m \geq(r-1)(r-3) /(8 r)$. Now, $q\left(m ; r^{2}\right)=h_{I(m ; n)+}\left(\alpha\left(m ; r^{2}\right)\right)$, and using $t=\alpha\left(m ; r^{2}\right)$ and assuming that $I\left(m ; r^{2}\right)^{+}$has minimal Hilbert function, we have $h_{I(m ; n)+}\left(\alpha\left(m ; r^{2}\right)\right)=\max \left\{0,(t+2)(t+1) / 2-\left(r^{2}-1\right) m(m+1) / 2-(m+1)(m+2) / 2\right\}$. But $(t+2)(t+1) / 2-\left(r^{2}-1\right) m(m+1) / 2-(m+1)(m+2) / 2 \leq 0$ for $r \geq 3$ with $m \geq\left(r^{2}-9\right) / 8$, and since $\left(r^{2}-9\right) / 8 \geq(r-1)(r-3) /(8 r)$ when $r \geq 3$, we see that maximal rank holds for $I\left(m ; r^{2}\right)$ if $m \geq\left(r^{2}-9\right) / 8$ for $r \geq 3$, whenever $I\left(m ; r^{2}\right)$ and $I\left(m ; r^{2}\right)^{+}$have minimal Hilbert function.

Our results on resolutions of uniform ideals in certain cases extend more generally. We present some such results now.

Proposition 3.3. Let $2 \leq r \leq n$ be integers and let $\mathbf{m}=\left(m_{1}, \ldots, m_{n}\right)$, where each $m_{i}$ is a nonnegative integer with $m=m_{1}=\cdots=m_{r}>0$. Assume for $r$ general points of $\mathbf{P}^{2}$ that $q(m ; r)=0$ and $\sum_{i>r}\left(m_{i}^{2}+m_{i}\right) / 2<h_{I(m ; r)}(\alpha(m ; r))$ and for $n$ general points of $\mathbf{P}^{2}$ that $I(\mathbf{m} ; n)$ has minimal Hilbert function. Then maximal rank holds for $I(\mathbf{m} ; n)$ for $n$ general points of $\mathbf{P}^{2}$.

Proof. By the hypothesis $\sum_{i>r}\left(m_{i}^{2}+m_{i}\right) / 2<h_{I(m ; r)}(\alpha(m ; r))$, we see that $\alpha(m ; r)$ $=\alpha(\mathbf{m} ; n)$; so $q(m ; r)=0$ implies $q(\mathbf{m} ; n)=0$, and the result follows by Lemma 2.4(b).

Note that if $r \geq 9$ in Proposition 3.3 then, as conjectured by QHC, we expect that $I(\mathbf{m} ; n)$ indeed has minimal Hilbert function if $\mathbf{m}$ is quasiuniform. Similarly, we expect that the hypotheses of minimality in the following result always hold (since after reordering $m_{i}$, if need be, for $i>r^{2}, \mathbf{m}$ is quasiuniform).

Corollary 3.4. Let $n \geq r^{2}$ where $r \geq 3$ is odd, let $m=m_{1}=\cdots=m_{r^{2}} \geq$ $\left(r^{2}-9\right) / 8 \geq \sum_{r^{2}<i \leq n}\left(m_{i}^{2}+m_{i}\right) / 2$ and let $\mathbf{m}=\left(m_{1}, \ldots, m_{n}\right)$. If $I(\mathbf{m} ; n)$ has minimal Hilbert function for $n$ general points of $\mathbf{P}^{2}$ and if $I\left(m ; r^{2}\right)$ and $I\left(m ; r^{2}\right)^{+}$ have minimal Hilbert function for $r^{2}$ general points of $\mathbf{P}^{2}$, then maximal rank holds for $I(\mathbf{m} ; n)$ for $n$ general points of $\mathbf{P}^{2}$.

Proof. As in the proof of Theorem 3.2(b), $I\left(m ; r^{2}\right)$ and $I\left(m ; r^{2}\right)^{+}$having minimal Hilbert function implies that $q\left(m ; r^{2}\right)=0$. From Lemma 2.5(ii) we find that $h_{I\left(m ; r^{2}\right)}\left(\alpha\left(m ; r^{2}\right)\right)=\left(r^{2}-1\right) / 8$; so Proposition 3.3 gives the result.

We close with a result and a remark involving no conditional hypotheses of minimality. 
Proposition 3.5. Let $m>0$, let $t \geq 0$, and choose $n \geq 9$ general points of $\mathbf{P}^{2}$ such that $-m-1 \leq n-[9+3 t m+(t+1)(t+2) / 2] \leq m$. Then maximal rank holds for $I(\mathbf{m} ; n)$ for $\mathbf{m}=\left(m_{1}, \ldots, m_{n}\right)$ with $m=m_{1}=\cdots=m_{9}$ and $m_{i}=1$ for $9<i \leq n$.

Proof. We first note that $I(m ; 9)$ has minimal Hilbert function. Indeed, for $I(m ; 9)$ to fail to have minimal Hilbert function, we would need to have

$$
h^{0}\left(X, \mathcal{O}_{X}\left(D_{t}\right)\right) h^{1}\left(X, \mathcal{O}_{X}\left(D_{t}\right)\right)>0 \text { for some } t>0,
$$

where $X$ is the blow-up of $\mathbf{P}^{2}$ at 9 general points and $D_{t}=t L-m\left(E_{1}+\cdots+E_{9}\right)$, as in Lemma 2.11 But by [Ha4, for example, this can occur only if $D_{t} \cdot A<0$ for some $(-1)$-curve $A$, and this is ruled out by Lemma 2.11. Replacing $D_{t}$ by $D_{t}^{+}$or $D_{t}^{-}$respectively, the same argument shows that $I(m ; 9)^{+}$and $I(m ; 9)^{-}$also have minimal Hilbert functions. Since simple points impose independent conditions, we now see that $I(\mathbf{m} ; n), I(\mathbf{m} ; n)^{+}$and $I(\mathbf{m} ; n)^{-}$all have minimal Hilbert functions.

Now, $\alpha(m ; 9)=3 m$ and $I(m ; 9)_{3 m}$ is 1-dimensional for $m>0$; so it is clear that maximal rank holds for $I(m ; 9)$. Thus, we may assume that $n>9$. But if $8+(3 t-1) m+(t+1)(t+2) / 2 \leq n \leq 8+3 t m+(t+1)(t+2) / 2$, we can easily check by minimality of the corresponding Hilbert functions that $\alpha(\mathbf{m} ; n)=3 m+t$ and then that $q(\mathbf{m} ; n)=0$; whereas, if $9+3 t m+(t+1)(t+2) / 2 \leq n \leq 9+(3 t+$ $1) m+(t+1)(t+2) / 2$, then $\alpha(\mathbf{m} ; n)=3 m+t+1$ and $l(\mathbf{m} ; n)>0$. Thus, the result follows by Lemma 2.4

Remark 3.6. The basic idea of Proposition 3.5 applies more generally to "fat points with tails". Given any sequence $\mathbf{m}=\left(m_{1}, m_{2}, \ldots, m_{r}\right)$ of positive integers (which for convenience we may as well assume is nonincreasing), consider the extended sequence $\mathbf{e}=\left(e_{1}, \cdots, e_{r+s}\right)$, where $e_{i}=m_{i}$ for $i \leq r$ and $e_{i}=1$ for $i>r$. Let $\tau(\mathbf{m} ; r)$ be the least $t$ such that, for general points $p_{i}, m_{1} p_{1}+\cdots+m_{r} p_{r}$ imposes independent conditions on forms of degree $t$. (For a discussion of various upper bounds for $\tau$, see [Ha8].) Since $\tau\left(\mathbf{m}^{+} ; r\right) \geq \tau(\mathbf{m} ; r), \tau\left(\mathbf{m}^{+} ; r\right) \geq \alpha(\mathbf{m} ; r)$ and $\alpha\left(\mathbf{m}^{+} ; r\right) \geq \alpha(\mathbf{m} ; r)$, and since simple points impose independent conditions, if $t \geq \tau\left(\mathbf{m}^{+} ; r\right)$ and $s \geq h_{I(\mathbf{m} ; r)}(t)$, then $\alpha(\mathbf{e} ; r+s)>t$; so $I\left(\mathbf{e}^{+} ; r+s\right)$ and $I(\mathbf{e} ; r+s)$ have minimal Hilbert functions. Also, $h_{I(\mathbf{m} ; r)}(t) \geq h_{I\left(\mathbf{m}^{-} ; r\right)}(t-1)$ and so $\alpha\left(\mathbf{e}^{-} ; r+s\right) \geq t$, but $\tau\left(\mathbf{m}^{+} ; r\right) \geq \tau\left(\mathbf{m}^{-} ; r\right)$ and so $I\left(\mathbf{e}^{-} ; r+s\right)$ has minimal Hilbert function, also. Now, if $h_{I(\mathbf{m} ; r)}(t) \leq s<h_{I(\mathbf{m} ; r)}(t+1)-\max \left(m_{1}+1, t-m_{1}+2\right)$, then $l(\mathbf{e} ; r+s)>0$ and $q(\mathbf{e} ; r+s)>0$; so maximal rank holds for $I(\mathbf{e} ; r+s)$ by Lemma 2.4(d). Also, if $h_{I(\mathbf{m} ; r)}(t+1)-\min \left(m_{1}+1, t-m_{1}+2\right) \leq s<h_{I(\mathbf{m} ; r)}(t+1)$, then $l(\mathbf{e} ; r+s)=0$ and $q(\mathbf{e} ; r+s)=0$; so now maximal rank holds for $I(\mathbf{e} ; r+s)$ by Lemma 2.4(a).

\section{Conjectures And Examples}

Many of our results in Section 3 employ certain assumptions about minimality on the Hilbert function. However, these assumptions are either known to be met or, as we discuss below, are conjectured to be.

The Hilbert function of $I(\mathbf{m} ; n)$ need not be minimal when $n \leq 9$, even in the uniform case, which makes behavior in this range somewhat complicated. In the case of quasiuniform ideals, as indicated by the following Quasiuniform Hilbert function Conjecture (QHC), it is expected that things are very simple for $n>9$ : 
Conjecture 4.1. (QHC) For $n>9$ general points of $\mathbf{P}^{2}, I(\mathbf{m} ; n), I(\mathbf{m} ; n)^{+}$and $I(\mathbf{m} ; n)^{-}$have minimal Hilbert function if $I(\mathbf{m} ; n)$ is quasiuniform.

Whereas resolutions are known (see $[\mathrm{FHH}])$ for ideals $I(\mathbf{m} ; n)$ involving $n \leq 8$ general points (and for uniform $\mathbf{m}$ when $n \leq 9$ [Ha6]), they remain unknown and, in general, unconjectured for $n>9$. Again, however, we expect things to be particularly simple for quasiuniform ideals for $n>9$ general points. In particular, we have the following Resolution Conjectures, URC and QRC; note that QRC implies URC, and that URC is equivalent to Conjecture 6.3 (Maximal Rank Conjecture) of [Ha6]:

\section{Conjecture 4.2.}

(a) (URC) For $n>9$ general points of $\mathbf{P}^{2}$, maximal rank holds for uniform ideals $I(m ; n)$ having minimal Hilbert function.

(b) (QRC) For $n>9$ general points of $\mathbf{P}^{2}$, maximal rank holds for quasiuniform ideals $I(\mathbf{m} ; n)$ with minimal Hilbert function.

Note that ideals $I(m ; n)$ with minimal Hilbert function for which maximal rank fails to hold can occur if $n<9$; take, for example, $m=9$ and $n=7$ (see Ha7, [Ha6 or $[\mathrm{FHH}]$ ). On the other hand, Example 4.6 gives various examples in which URC is, based on our results, now known to hold. Currently, much less can be said in support of QRC, but the end of Section 3 gives some supporting evidence.

Whereas it is unclear how QRC might be extended in general to the nonquasiuniform case, QHC is, as we show in Remark 4.4 a special case of Conjecture 4.3. which makes no assumptions on the uniformity of the multiplicities $m_{i}$. For other equivalent variants of Conjecture 4.3, see [Ha1, [Hi], Gi1], [Ha3]; for a nice survey, see [Mi] or $\mathrm{Ha}$ ].

Conjecture 4.3. For each positive integer $d$, there is a nonempty open set $U_{d}$ of points $\left(p_{1}, \ldots, p_{n}\right) \in\left(\mathbf{P}^{2}\right)^{n}$ such that on the surface $X$ obtained by blowing up $p_{1}, \ldots, p_{n}$ we have the following (where $L$ is the total transform of a line):

(i) either $h^{0}\left(X, \mathcal{O}_{X}(F)\right)=0$ or $h^{1}\left(X, O_{X}(F)\right)=0$ for any numerically effective divisor $F$ with $F \cdot L \leq d$;

(ii) if $C$ is a prime divisor on $X$ of negative self-intersection with $C \cdot L \leq d$, then $C^{2}=C \cdot K_{X}=-1$.

Remark 4.4. Using the method of proof of Corollary 5.6 of [Ha6], we now show that Conjecture 4.3 implies Conjecture 4.1 First, $I(\mathbf{m} ; n)_{t}$ corresponds for each $t$ to the complete linear system $\left|D_{t}\right|$ on $X$ of the divisor $D_{t}=t L-m_{1} E_{1}-\cdots-m_{n} E_{n}$, where $X \rightarrow \mathbf{P}^{2}$ is the blow-up of the points $p_{1}, \ldots, p_{n}, L$ is the total transform to $X$ of a line in $\mathbf{P}^{2}$, and each $E_{i}$ is the exceptional locus of the blow-up of $p_{i}$. If $\left|D_{t}\right|$ is nonempty and $A$ is a reduced irreducible divisor with $D_{t} \cdot A<0$, then $A^{2}<0$ and $L \cdot A \leq t$. Hence, $A^{2}=A \cdot K_{X}=-1$ by Conjecture 4.3, so $D_{t}$ is numerically effective and thus $I\left(\left(m_{1}, \ldots, m_{n}\right) ; n\right)$ has minimal Hilbert function by Conjecture 4.3. The same argument applying Lemma 2.11 works for $I\left(\mathbf{m}^{+} ; n\right)_{t}$ using $D_{t}^{+}$and, when $m_{1}>0$, for $I\left(\mathbf{m}^{-} ; n\right)_{t}$ using $D_{t}^{-}$.

Remark 4.5. As an aside, we mention how by assuming Conjecture 4.3 it is a simple matter to explicitly compute the Hilbert function of an ideal $I(\mathbf{m} ; n)$. Using the notation of Remark 4.4 one subtracts from $D_{t}$ irreducible effective divisors $C$ satisfying $C \cdot L \leq t$ and $C^{2}=C \cdot K_{X}=-1$ (all such $C$ are known by [N2]) 
for which $C \cdot D_{t}<0$. Eventually, a divisor $D$ is obtained from $D_{t}$ that either meets all such $C$ nonnegatively or that has $D \cdot L<0$. In the latter case, $|D|$ is empty and $h^{0}\left(X, \mathcal{O}_{X}(D)\right)=0$. In the former case, we may assume $D \cdot L \geq 0$; so $h^{2}\left(X, \mathcal{O}_{X}(D)\right)=0$ by duality, but Conjecture 4.3 (ii) implies that either $|D|$ is empty or $D$ is numerically effective. So by Conjecture 4.3 (i) and Riemann-Roch, we can compute $h^{0}\left(X, \mathcal{O}_{X}(D)\right)$ and hence the Hilbert function of $I(\mathbf{m} ; n)$.

Example 4.6. For $r^{2}=16$ general points of $\mathbf{P}^{2}$ in any characteristic, [N1] shows that $\alpha(m ; n)>4 m$; so it follows from Lemma 2.5(i) that $\alpha(m ; n)=r m+r / 2-1$ for each $m>0$ for $r=4$. Thus, maximal rank holds for $I(m ; n)$ in all characteristics by Theorem 3.2 for all $m \geq 0$ if $n=16$. Additionally, in characteristic 0 by [CM1] and CM2], $I(m ; n)$ has minimal Hilbert function for any $n>9$ general points when $m \leq 12$. Consequently, by Theorem 3.2(a) and Lemma 2.5(i), maximal rank holds for $I\left(m ; r^{2}\right)$ in characteristic 0 whenever $12 \geq m \geq(r-2) / 4$ with $r>3$ and $r$ even. In particular, this includes any $n$ that is an even square in the range $16<n \leq 50^{2}=2500$ when $m=12$; any even square $16<n \leq 46^{2}=2116$ with $m=11 ; \ldots$; and any even square $16<n \leq 14^{2}=196$ with $m=3$. (The case that $m=2$ with any $n>9$ is done by $[\mathrm{Id}]$.)

Further interesting examples are given whenever $n$ is such that $n(m+1) m / 2=$ $(t+1) t / 2$ for some $t$. For example, we can take $n=s^{2} m(m+1)+s$ for some $s$. For more examples, define a sequence $b_{i}$ via $b_{i+1}=\left(b_{i}-1\right)^{2}$, where $b_{0}$ is any integer bigger than 2. For any $i$, take $n=b_{i}\left(b_{i}-1\right) /\left(b_{0}\left(b_{0}-1\right)\right)$. This is an integer, since $b_{i}\left(b_{i}-1\right)$ divides $b_{i+1}\left(b_{i+1}-1\right)$ for every $i$. If we take $b_{0}=m+1$ for some $m>1$, then clearly $n(m+1) m / 2=(t+1) t / 2$ for $t=b_{i}-1$.

If $n(m+1) m / 2=(t+1) t / 2$ for some $t$ and if $\alpha=\alpha(m ; n)$ and thus the regularity of $I(m ; n)$ were to have their expected values, then $I(m ; n)$ would have no generators in degrees beyond $\alpha$, in which case the resolution would be $0 \rightarrow R[-\alpha-1]^{\alpha} \rightarrow$ $R[\alpha]^{\alpha+1} \rightarrow I(m ; n) \rightarrow 0$, as conjectured by the URC. By the asymptotic result of $[\mathrm{AH}$, fixing $m>2$, it follows that the regularity of $I(m ; n)$ is indeed $t$ for $n$ sufficiently large, and thus maximal rank holds for $I(m ; n)$. Alternatively, by CM2 it follows that maximal rank holds for $I(m ; n)$ in characteristic 0 for any $n$ and $m$ such that $n(m+1) m / 2=(t+1) t / 2$ for some $t$, as long as $n \geq 10$ and $m \leq 12$.

In the case of ideals $I(m ; n)$ for which maximal rank holds and for which $n(m+$ 1) $m / 2=(t+1) t / 2$, the multiplication map $\mu_{\alpha}: I(m ; n)_{\alpha} \otimes R_{1} \rightarrow I(m ; n)_{\alpha+1}$ is surjective. We can also apply our results with $[\mathrm{AH}]$ to give additional examples for which $\mu_{\alpha}$ is injective. By [AH], for each $m$ and all $n$ sufficiently large, $I(m ; n)$ and $I(m ; n)^{+}$both have minimal Hilbert function. Thus, $q(m ; n)=\max (0,(t+2)(t+$ $1) / 2-n m(m+1) / 2-m-1)$, where $t=\alpha(m ; n)$ is the least positive integer with $(t+2)(t+1) / 2-n m(m+1) / 2>0$. By Lemma 2.4(b), maximal rank holds for $I(m ; n)$ if $q(m ; n)=0$ (in which case $\mu_{\alpha(m ; n)}$ is injective). Thus, given $m$ and any $a>0$ with $a(a+1)<2(m+1)$, it is enough to find infinitely many $n$ such that $(t+2)(t+1)=n m(m+1)+a(a+1)$ for some $t$.

We now show that there are infinitely many such $n$ as long as $m>2$. More generally, for each $m>2$ and $a \geq 0$, there are infinitely many $n$ such that $(t+$ $2)(t+1)=n m(m+1)+a(a+1)$ for some $t$. We saw that there are infinitely many $n^{\prime}$ such that there is an $s$ with $s^{2}+s=n^{\prime} m(m+1)$. Taking $t+1=(s-a)^{2}-a^{2}-a-1$, we have $(t+2)(t+1)-a(a+1)=(t+a+2)(t-a+1)=\left((s-a)^{2}-a^{2}\right)\left((s-a)^{2}-(a+1)^{2}\right)=$ $(s-2 a) s(s+1)(s-2 a-1)=n m(m+1)$, where $n=(s-2 a)(s-2 a-1) n^{\prime}$. 
We finish by giving the proof of Corollary 1.1

Proof of Corollary 1.1. (a) For $m=1$, see GGR; for $m=2$, see [Id]. For $m>2$, use Example 4.6 where we saw for each $m$ that there is an infinite set of $n$ for which maximal rank holds for $I(m ; n)$.

For (b), by [E] and Lemma 2.5(i), it follows that $\alpha(m ; n)=m r+r / 2-1$ for $m \geq(r-2) / 4$ when $n=4^{s}$. Now apply Theorem 3.2(a), using Lemma 2.5)(i) to compute $h$.

\section{REFERENCES}

[AH] J. Alexander and A. Hirschowitz. An asymptotic vanishing theorem for generic unions of multiple points, Invent. Math. 140 (2000), no. 2, 303-325. MR 2001i:14024

[Cam] G. Campanella. Standard bases of perfect homogeneous polynomial ideals of height 2, J. Algebra 101 (1986), 47-60. MR 87f:13005

[Cat] M. V. Catalisano. "Fat" points on a conic, Comm. Algebra 19 (1991), no. 8, 2153-2168. MR 93b:14016

[CM1] C. Ciliberto and R. Miranda. Degenerations of planar linear systems, J. Reine Angew. Math. 501 (1998), 191-220. MR 2000m:14005

[CM2] C. Ciliberto and R. Miranda. Linear systems of plane curves with base points of equal multiplicity, Trans. Amer. Math. Soc. 352 (2000), 4037-4050. MR 2000m:14006

[DGM] E. D. Davis, A. V. Geramita, and P. Maroscia. Perfect homogeneous ideals: Dubreil's theorems revisited, Bull. Sci. Math. (2) 108 (1984), 143-185. MR 86m:13024

[Du] P. Dubreil. Sur quelques propriétés des systèmes de points dans le plan et des courbes gauches algébriques, Bull. Soc. Math. France, 61 (1933), 258-283.

[E] L. Evain. La fonction de Hilbert de la réunion de $4^{h}$ gros points génériques de $\mathbf{P}^{2}$ de même multiplicité, J. Algebra Geom. 8 (1999), 787-796. MR 2000e:13023]

[F1] S. Fitchett. On bounding the number of generators for fat point ideals on the projective plane, J. Algebra 236 (2001), 502-521. MR 2002c:13028

[F2] S. Fitchett. Maps of linear systems on blow ups of the projective plane, J. Pure and Applied Algebra 156 (2001), 1-14. MR 2002c:14013

$[\mathrm{FHH}]$ S. Fitchett, B. Harbourne and S. Holay. Resolutions of fat point ideals involving eight general points of $\mathbf{P}^{2}$, J. Algebra 244 (2001), 684-705. MR 2002g:14089

[GGR] A. V. Geramita, D. Gregory and L. Roberts. Monomial ideals and points in projective space, J. Pure and Appl. Algebra 40 (1986), 33-62. MR 87d:13023

[GO] A. V. Geramita, and F. Orrechia. Minimally generating ideals defining certain tangent cones, J. Algebra 78 (1982), 36-57. MR 84e:13028

[Gi1] A. Gimigliano. Our thin knowledge of fat points, Queen's papers in Pure and Applied Mathematics, no. 83, The Curves Seminar at Queen's, vol. VI (1989). MR 91a:14007

[Gi2] A. Gimigliano. Regularity of linear systems of plane curves, J. Algebra 124 (1989), 447460. MR 90i:14005

[Ha1] B. Harbourne. The geometry of rational surfaces and Hilbert functions of points in the plane, Canad. Math. Soc. Conf. Proc. 6 (1986), 95-111. MR 87k:14041

[Ha2] B. Harbourne. Iterated blow-ups and moduli for rational surfaces, in: Algebraic Geometry, Sundance 1986, Lecture Notes in Math., 1311 (1988), 101-117. MR 90b:14009

[Ha3] B. Harbourne. Points in Good Position in $\mathbf{P}^{2}$, in: Zero-dimensional schemes, Proceedings of the International Conference held in Ravello, Italy, June 8-13, 1992, De Gruyter, 1994. MR 95m:14005

[Ha4] B. Harbourne. Anticanonical rational surfaces, Trans. Amer. Math. Soc. 349 (1997), 11911208. MR 97f:14007

[Ha5] B. Harbourne. Free Resolutions of Fat Point Ideals on $\mathbf{P}^{2}$, J. Pure and Applied Algebra 125 (1998), 213-234. MR 99d:13016

[Ha6] B. Harbourne. The ideal generation problem for fat points, J. Pure and Applied Algebra 145 (2000), 165-182. MR 2001c:13028

[Ha7] B. Harbourne. An Algorithm for Fat Points on $\mathbf{P}^{2}$, Canad. J. Math. 52 (2000), 123-140. MR 2001g:13037 
[Ha8] B. Harbourne. Problems and Progress: A survey on fat points in $\mathbf{P}^{2}$, Queen's papers in Pure and Applied Mathematics, The Curves Seminar at Queen's, vol. 123, 2002. CMP 2002:12

[HR] B. Harbourne and J. Roé. Linear systems with multiple base points in $\mathbf{P}^{2}$, preprint (2001).

[Hi] A. Hirschowitz. Une conjecture pour la cohomologie des diviseurs sur les surfaces rationelles génériques, Journ. Reine Angew. Math. 397 (1989), 208-213. MR 90g:14021

[Id] M. Idà. The minimal free resolution for the first infinitesimal neighborhoods of $n$ general points in the plane, J. Algebra 216 (1999), 741-753. MR 2000d:13022

[Mi] R. Miranda. Linear Systems of Plane Curves, Notices Amer. Math. Soc. 46(2) (1999), 192-202. MR 99m:14012

[Mu D. Mumford. Varieties defined by quadratic equations, in: Questions on Algebraic Varieties, Corso C.I.M.E. 1969 Rome: Cremonese, 1970, 29-100. MR 44:209

[N1] M. Nagata. On the 14th problem of Hilbert, Amer. J. Math. 81 (1959), 766-772. MR 21:4151

[N2] M. Nagata. On rational surfaces, II, Mem. Coll. Sci. Univ. Kyoto, Ser. A Math. 33 (1960), 271-293. MR 23:A3740

[R] J. Roé. Linear systems of plane curves with imposed multiple points, Illinois J. Math. 45 (2001), 895-906.

[X] G. Xu. Ample line bundles on smooth surfaces, Jour. Reine Angew. Math. 469 (1995), 199-209. MR 96k:14003

Department of Mathematics and Statistics, University of Nebraska-Lincoln, LinCOLn, NeBraska 68588-0323

E-mail address: bharbour@math.unl.edu

$U R L:$ http://www. math.unl.edu/ bharbour/

Department of Mathematics, Southeast Community College, Lincoln, Nebraska 68508

E-mail address: sholay@southeast.edu

Florida Atlantic University, Honors College, Jupiter, Florida 33458

E-mail address: sfitchet@fau.edu 